\title{
Instituições de Ensino Superior no ranking Times Higher Education: uma análise sobre ensino, pesquisa e renda industrial
}

\section{Higher Education Institutions in Times Higher Education ranking: an analysis of teaching, research and industrial income}

\author{
Luiz Gustavo Cordeiro \\ Universidade Tecnológica Federal do Paraná - UTFPR - Ponta Grossa - Brasil \\ gucordeiro@gmail.com \\ Caroline Lievore \\ Universidade Tecnológica Federal do Paraná - UTFPR - Ponta Grossa - Brasil \\ carolinevore1@gmail.com \\ Regina Negri Pagani \\ Universidade Tecnológica Federal do Paraná - UTFPR - Ponta Grossa - Brasil \\ reginapagani@utfpr.edu.br
}

\section{Resumo}

Os rankings universitários são ferramentas que surgiram por meio do fenômeno da globalização a fim de comparar a performance das Instituições de Ensino Superior (IES). O presente trabalho busca identificar as universidades mais bem ranqueadas no ranking Times Higher Education nos fatores "Ensino", "Pesquisa" e "Renda industrial" na avaliação de 2020. Os resultados apontam uma disparidade geográfica entre os indicadores Ensino, Pesquisa e Renda Industrial. Nos dois primeiros indicadores, Ensino e Pesquisa, há uma concentração de instituições nos Estados Unidos e no Reino Unido e uma distribuição homogênea no indicador "renda industrial". As análises evidenciaram que apenas uma das universidades selecionadas na pesquisa está presente nos três indicadores, a universidade chinesa de Tsingua. Conclui-se que a autonomia financeira das instituições pode ser determinante para o melhor posicionamento dos critérios analisados no ranking. Palavras-chave: Times Higher Education, transferência de tecnologia, instituições de ensino superior.

\footnotetext{
Abstract

University rankings are tools that emerged through the phenomenon of globalization in order to compare the performance of Higher Education Institutions (HEls). This paper aims to identify the best ranked universities in the Times Higher Education ranking by the factors "Teaching", "Research" and "Industrial Income" in the 2020 assessment. In the first two indicators, Teaching and Research, there is a concentration of institutions from the United States and United Kingdom and a homogeneous distribution in the indicator "industrial income". The analysis showed that only one of the universities selected in the research that is present in the three indicators, the Chinese University of Tsingua. It is concluded that the instituions financial autonomy can be determinant for the best positioning of the criteria analyzed by the ranking.
} 
Keywords: Times Higher Education; technology transfer; higher education institutions.

\section{Introdução}

O fenômeno da globalização, acarretou a transição das economias baseadas principalmente em propriedades industriais para economias baseadas em propriedades intelectuais, tais como pesquisas e patentes desenvolvidas dentro das IES (OLSEN; PETERS, 2007). Tal mudança, de foco econômico, acarretou também mudanças culturais e gerenciais no sistema de ensino superior internacional, incitando a criação de indicadores ligados ao ensino superior e uma classificação a nível global, tais como os rankings universitários (BASTEDO; BOWMAN,2010; DELGADO-MÁRQUEZ; HURTADOTORRES 2011). Os rankings universitários globais, tiveram início em 2003, com o propósito de comparar as universidades em escala global, primeiramente com 0 Academic Ranking of World Universities (ARWU), criado pela Shanghai Jiao Tong University Institute of Higher Education, seguido pelo Times Higher Education Supplement (THES) em 2004, originado da parceria entre a Thomson Reuters e a Qualquarelli Symonds Ltd. (BASTEDO; BOWMAN, 2010; DOGAN e AL, 2018).

$O$ ranking THES, foi criado para obter um alcance maior do que o ranking ARWU, em relação aos indicadores de desempenho (Wong; Singh, 2009), sendo composto por cinco fatores base: ensino, pesquisa, citações, renda industrial e perspectiva internacional. A parceria entre THE e QS se desfez em 2010, dando origem a dois rankings, o Times Higher Education World Universities Ranking (THE-WU) e QS - World University Rankings (QA-WU), sendo que o THE-WU manteve seus cinco critérios de avaliação. Jeremic e Jovanivic-Milenkovic (2014), apontam que o ranking THE foi um dos primeiros a dar o mesmo peso para o critério de ensino, citações e publicações, e a avaliar a habilidade de geração de inovações, invenções e consultorias através do critério "renda industrial".

Marginson (2007) cita que, mesmo em seus primeiros anos de atividade, os rankings universitários redefiniram o contexto global do ensino superior, expondo as IES à uma espécie de competição. Cheng, Wang e Liu (2014), denotam que tal competição faz com que as IES busquem melhores colocações e sejam consideradas "World-Class University" (WCU). Marginson (2014) faz uma crítica dizendo que os esforços para tornar uma IES uma WCU também são ligados ao desejo de estima tanto do corpo docente, que deseja ser associado a uma instituição de renome, quanto para o corpo discente que deseja estudar em uma instituição mundialmente reconhecida. Complementando Jeremic e Jovanivic-Milenkovic (2014) indagam que o crescente número de métricas para avaliar as IES, é, por vezes, usado como guia para comunidade acadêmica estabelecer critétios no ensino superior como um todo, visto que, os rankings podem ser importantes ferramentas de marketing. Já Foster (2018), cita que para atingir o status de WCU as IES devem se atentar a distribuição de seus investimentos, distribuindo-os de acordo com os critérios avaliados no ranking.

Montesinos et al. (2008) apontam que, entre as missões das WCU, estão o ensino, a pesquisa e a transferência de tecnologia, e salienta a falta de indicadores específicos que atendam a terceira missão das universidades, a transferência de tecnologia. Da mesma forma Perez-Esparrels e Orduna-Malea (2018), denotam que as métricas do ranking THE-WU apesar de avaliarem questões de transferência de tecnologia e conhecimento através do fator "renda industrial", não atribuem valor suficiente para que tal fator possa ser determinante para a classificação geral do ranking.

Os rankings unversitários são analisados em diversas pesquisas. Entre as publicações no tema, Laughton (2003); Kroth e Daniel (2008); Wong e Singh (2009); Soh (2013); Baty (2014); Valmorbida, Cardoso e Ensslin (2015); Hayes (2017); Sabata e 
Cadeñosa (2017); Perez-Esparrels e Orduna-Malea (2018) discutem as métricas utilizadas e suas aplicabilidades de acordo com contextos específicos dos diferentes modelos de IES. Em outro âmbito, Rosselot (2005); Deem, Mok e Lucas (2008); Rodionov, Rudskaia e Kushneva (2014); Thien e Ong (2016); Liu, Moshi e Awuor (2019), usam os rankings universitários como base para a internacionalização das instituições, permitindo assim que as mesmas galguem o título de WCU. E alguns estudos evidenciam as barreiras culturais, religiosas e políticas de países que buscam a internacionalização de suas pesquisas e instituições (RAHIM, 2012; HAYES, 2016; FORSTER, 2017; AL KWAIT; DOWNING; SUBBARAYALU, 2019).

Há uma lacuna na literatura de estudos que avaliem as IES ranqueadas, fazendo alguma correlação entre os indicadores de ensino, pesquisa e renda industrial propostos pelos rankings. Deste modo, esta pesquisa tem como objetivo identificar as universidades mais bem ranqueadas no THE nos fatores "Ensino", "Pesquisa" e "Renda industrial" na avaliação de 2020.

\section{Metodologia}

A pesquisa é exploratória e documental, visto que são retiradas informações do ranking THE de 2020. Segundo Silva et al. (2009), a pesquisa documental é um método em que não apenas os documentos escolhidos devem responder as questões da pesquisa, mas também as análises, que podem obter diversas interpretações dentro de um mesmo documento, a depender do foco da pesquisa realizada.

Para atender o objetivo da pesquisa foram comparados os dados apresentados pelas quinze universidades melhor ranqueadas no índice geral do THE-WU de 2020, que classifica 1.396 universidades de 92 países. Em seguida, foram analisadas estas quinze instituições, segundo cada um dos três indicadores que apresentam relação direta com as missões universitárias, o Ensino, a Pesquisa e a Transferência de Tecnologia, sendo estes indicadores do Ensino, Pesquisa e Renda Industrial. As métricas utilizadas para a classificação do ranking são apresentadas na Tabela 1.

Tabela 1: Métricas usadas pelo ranking THE-WU

\begin{tabular}{ccc}
\hline Fatores & Peso & Critérios \\
\hline Ensino & $30 \%$ & $\begin{array}{c}\text { Pesquisa de reputação: } 15 \% \\
\text { Proporção entre funcionários e } \\
\text { alunos: } 4,5 \%\end{array}$ \\
& & $\begin{array}{c}\text { Relação doutorado-bacharel: } 2,25 \% \\
\text { Proporção de doutores por membros } \\
\text { do corpo docente: } 6 \% \\
\text { Renda institucional: } 2,25 \%\end{array}$ \\
Pesquisa & $30 \%$ & $\begin{array}{c}\text { Pesquisa de reputação: } 18 \% \\
\text { Renda destinada à pesquisa: } 6 \% \\
\text { Produtividade em pesquisa: } 6 \% \\
\text { Renda }\end{array}$ \\
Industrial & $2,5 \%$ & $\begin{array}{c}\text { Capacidade de geração de inovações } \\
\text { para a indústria por parte das } \\
\text { universidades: } 2,5 \%\end{array}$ \\
\hline Fonte: Adaptado de Times Higher Education (2019)
\end{tabular}

Em seguida analisou-se a literatura e os documentos oficiais das próprias instituições, a fim de identificar possíveis fatores políticos, sociais e administrativos determinantes para a colocação das melhores IES em cada um dos indicadores selecionados. Por fim, foram realizados mapas gráficos para demonstrar visualmente a distribuição das instituições globalmente. 


\section{Resultados e discussões}

O ranking THE-WU avalia 1.396 universidades em 92 países. As universidades submetem seus dados para a análise de acordo com os critérios medidos pelo ranking. A Tabela 2, apresenta os países que foram analisados pelo ranking em 2020 de acordo com o número de IES e o percentual de instituições de cada país em relação ao total avaliado.

Tabela 2: Instituições avaliadas pelo ranking THE-WU 2020

\begin{tabular}{|c|c|c|c|c|c|}
\hline Países & $\begin{array}{l}\text { Quantida } \\
\text { de de IES }\end{array}$ & $\begin{array}{c}\text { Porcentagem } \\
\text { total }\end{array}$ & Países & $\begin{array}{l}\text { Quantidad } \\
\text { e de IES }\end{array}$ & $\begin{array}{c}\text { Porcentagem } \\
\text { total }\end{array}$ \\
\hline Estados Unidos & 172 & $12,3209 \%$ & Ucrânia & 6 & $0,4298 \%$ \\
\hline Japão & 110 & $7,8797 \%$ & Jordânia & 5 & $0,3582 \%$ \\
\hline Reino Unido & 100 & $7,1633 \%$ & Noruega & 5 & $0,3582 \%$ \\
\hline China & 81 & $5,8023 \%$ & Argentina & 4 & $0,2865 \%$ \\
\hline India & 56 & $4,0115 \%$ & Marrocos & 4 & $0,2865 \%$ \\
\hline Alemanha & 48 & $3,4384 \%$ & Nigéria & 4 & $0,2865 \%$ \\
\hline Brasil & 46 & $3,2951 \%$ & Eslováquia & 4 & $0,2865 \%$ \\
\hline Itália & 45 & $3,2235 \%$ & $\begin{array}{l}\text { Emirados Árabes } \\
\text { Unidos }\end{array}$ & 4 & $0,2865 \%$ \\
\hline Espanha & 45 & $3,2235 \%$ & Estônia & 3 & $0,2149 \%$ \\
\hline Irã & 40 & $2,8653 \%$ & Letônia & 3 & $0,2149 \%$ \\
\hline Rússia & 39 & $2,7937 \%$ & Líbano & 3 & $0,2149 \%$ \\
\hline França & 38 & $2,7221 \%$ & Lituânia & 3 & $0,2149 \%$ \\
\hline Taiwan & 36 & $2,5788 \%$ & Vietnã & 3 & $0,2149 \%$ \\
\hline Austrália & 35 & $2,5072 \%$ & Croácia & 2 & $0,1433 \%$ \\
\hline Turquia & 34 & $2,4355 \%$ & Chipre & 2 & $0,1433 \%$ \\
\hline Coréia do Sul & 31 & $2,2206 \%$ & Islândia & 2 & $0,1433 \%$ \\
\hline Canadá & 30 & $2,1490 \%$ & Iraque & 2 & $0,1433 \%$ \\
\hline Egito & 20 & $1,4327 \%$ & Cazaquistão & 2 & $0,1433 \%$ \\
\hline Chile & 18 & $1,2894 \%$ & Macau & 2 & $0,1433 \%$ \\
\hline $\begin{array}{l}\text { República } \\
\text { Tcheca }\end{array}$ & 17 & $1,2178 \%$ & Peru & 2 & $0,1433 \%$ \\
\hline México & 17 & $1,2178 \%$ & Filipinas & 2 & $0,1433 \%$ \\
\hline Tailândia & 16 & $1,1461 \%$ & Singapura & 2 & $0,1433 \%$ \\
\hline Paquistão & 14 & $1,0029 \%$ & Eslovênia & 2 & $0,1433 \%$ \\
\hline Polônia & 14 & $1,0029 \%$ & Sri Lanka & 2 & $0,1433 \%$ \\
\hline Malásia & 13 & $0,9312 \%$ & Venezuela & 2 & $0,1433 \%$ \\
\hline Países Baixos & 13 & $0,9312 \%$ & Bangladesh & 1 & $0,0716 \%$ \\
\hline Portugal & 13 & $0,9312 \%$ & Bielorrúsia & 1 & $0,0716 \%$ \\
\hline Suécia & 12 & $0,8596 \%$ & Brunei & 1 & $0,0716 \%$ \\
\hline Austria & 11 & $0,7880 \%$ & Bulgária & 1 & $0,0716 \%$ \\
\hline Suíça & 11 & $0,7880 \%$ & Costa Rica & 1 & $0,0716 \%$ \\
\hline Grécia & 10 & $0,7163 \%$ & Cuba & 1 & $0,0716 \%$ \\
\hline Africa do Sul & 10 & $0,7163 \%$ & Geórgia & 1 & $0,0716 \%$ \\
\hline Finlândia & 9 & $0,6447 \%$ & Gana & 1 & $0,0716 \%$ \\
\hline Irlanda & 9 & $0,6447 \%$ & Jamaica & 1 & $0,0716 \%$ \\
\hline Romênia & 9 & $0,6447 \%$ & Quênia & 1 & $0,0716 \%$ \\
\hline Algeria & 8 & $0,5731 \%$ & Kuwait & 1 & $0,0716 \%$ \\
\hline Bélgica & 8 & $0,5731 \%$ & Luxenburgo & 1 & $0,0716 \%$ \\
\hline Colômbia & 8 & $0,5731 \%$ & Malta & 1 & $0,0716 \%$ \\
\hline Hungria & 8 & $0,5731 \%$ & Montenegro & 1 & $0,0716 \%$ \\
\hline Nova Zelândia & 8 & $0,5731 \%$ & Nepal & 1 & $0,0716 \%$ \\
\hline Dinamarca & 7 & $0,5014 \%$ & Norte do Chipre & 1 & $0,0716 \%$ \\
\hline Arábia Saudita & 7 & $0,5014 \%$ & Omã & 1 & $0,0716 \%$ \\
\hline Hong Kong & 6 & $0,4298 \%$ & Porto Rico & 1 & $0,0716 \%$ \\
\hline Indonésia & 6 & $0,4298 \%$ & Catar & 1 & $0,0716 \%$ \\
\hline Israel & 6 & $0,4298 \%$ & Tanzânia & 1 & $0,0716 \%$ \\
\hline Tunísia & 6 & $0,4298 \%$ & Uganda & 1 & $0,0716 \%$ \\
\hline
\end{tabular}

Fonte: Autores (2019) 
Verifica-se que cerca de $12 \%$ das universidades avaliadas pelo ranking são de origem estadunidense, seguido do Japão com 7,8\% e Reino unido com 7,1\%. Em sétimo lugar com 3,3\% das instituições, está o Brasil.

Os dados são apresentados de forma gráfica na Figura 1, sendo que a tonalidade de azul é intensificada de acordo com a densidade dos dados distribuídos pelos países apresentados pela tabela anterior.

Figura 1: Distribuição Geográfica das Instituições presentes no ranking THE 2020

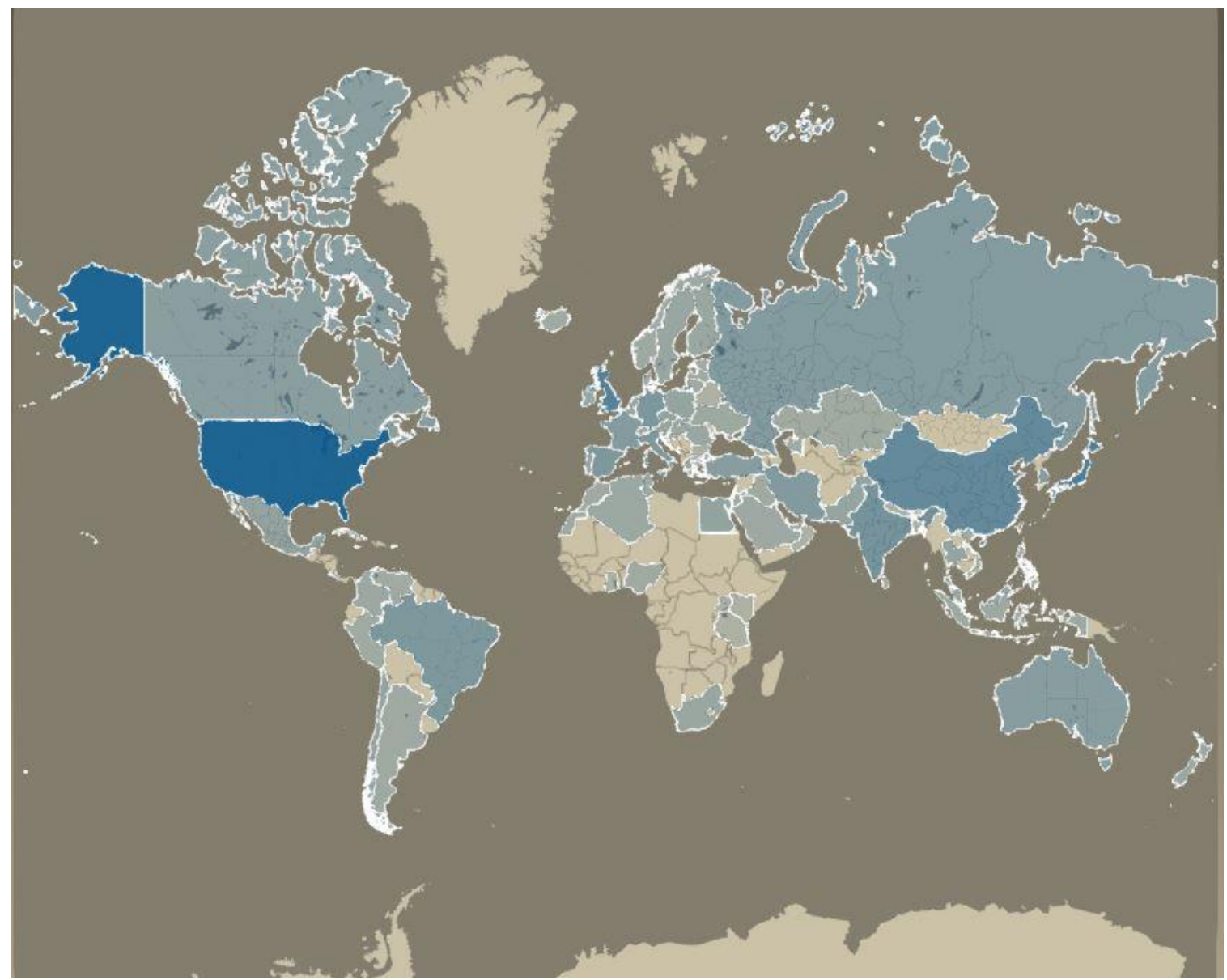

Fonte: Autores (2019)

É possível verificar que as instituições representadas no ranking THE-WU 2020 se apresentam em maior número em países como os Estados Unidos, Reino Unido, Japão e China. Fica também visível a falta de representantes de países do continente Africano, da Ásia e da América Latina.

A Tabela 3 apresentada as quinze universidades mais bem ranqueadas segundo a classificação geral do THE-WU 2020:

Tabela 3: Instituições mais bem ranqueadas segundo o ranking geral

\begin{tabular}{cccc}
\hline Ranking & Universidade & \multicolumn{1}{c}{ País } & Pontuação \\
\hline 1 & University of Oxford & Reino Unido & 95.4 \\
2 & California Institute of Technology & Estados Unidos & 94.5 \\
3 & University of Cambridge & Reino Unido & 94.4 \\
4 & Stanford University & Estados Unidos & 94.3 \\
5 & Massachusetts Institute of & Estados Unidos & 93.6 \\
6 & Technology & & 93.2 \\
7 & Princeton University & Estados Unidos & 93.0 \\
8 & Harvard University & Estados Unidos & 91.7 \\
9 & Yale University & Estados Unidos & 90.2
\end{tabular}




\begin{tabular}{lccc}
10 & Imperial College London & Reino Unido & 89.8 \\
11 & University of Pennsylvania & Estados Unidos & 89.6 \\
12 & Johns Hopkins University & Estados Unidos & 89.2 \\
13 & University of California, Berkeley & Estados Unidos & 88.3 \\
13 & ETH Zurich & Suiça & 88.3 \\
15 & UCL & Reino Unido & 87.1 \\
\hline
\end{tabular}

Fonte: Adaptado de Times Higher Education (2019)

Nota-se um grande número de instituições estadunidenses (cerca de 73,33\%), seguido de universidades do Reino Unido (20\%) e por fim uma universidade suíça, o ETH Zurich (representando 6,66\%). Oxford lidera o ranking geral, e parte desta liderança se deve as parcerias de pesquisa com outras universidades renomadas (The Telegraph, 2019). A Figura 2, apresenta de maneira ilustrativa a distribuição geográfica das instituições apresentadas na Tabela 2:

Figura 2: Distribuição Geográfica das melhores Instituições ranqueadas no ranking geral

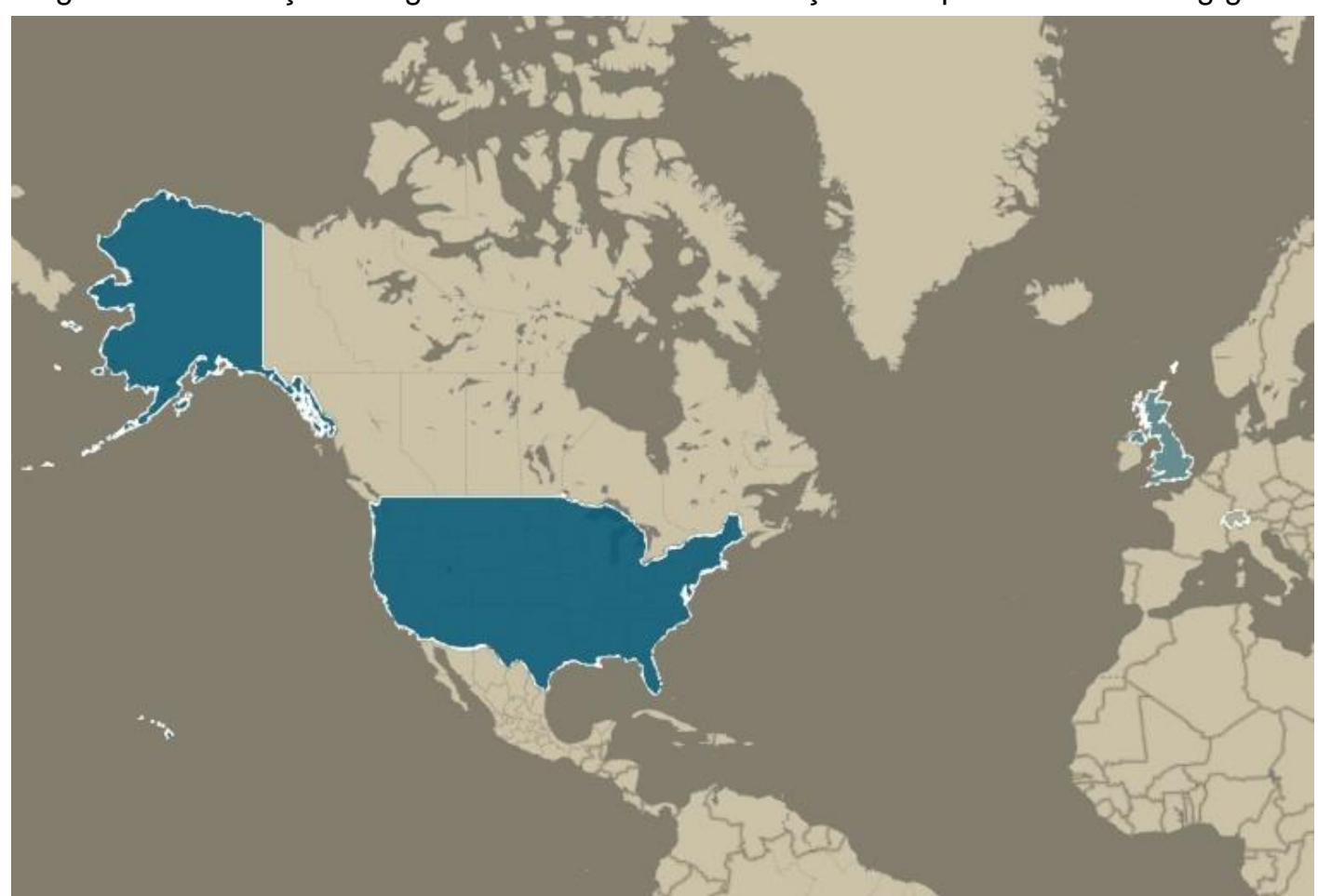

Fonte: Autores (2019)

É possível visualizar uma hegemonia do eixo Reino Unido e Estados Unidos, sendo apenas uma universidade de origem diferente, localizada na Suíça. Heyneman e Lee (2013), abordam que as universidades estadunidenses e inglesas, por possuírem renome internacional, geralmente se distribuem em primeiras colocações dos rankings, pois seu status Ihes garante a captação de talentos tanto nacionais, quanto internacionais. Além disso, os autores apontam que a maior parte das universidades dos Estados Unidos e Reino Unido possuem autonomia governamental, por serem instituições privadas ou semiprivadas que, segundo Etzkowitz et al. (2000) não dependem inteiramente da burocracia governamental, assim ganhando flexibilidade nas tomadas de decisões. Dentre as representadas no gráfico, $73,33 \%$ das instituições são privadas ou semiprivadas (estadunidenses e britânicas) enquanto 26,67\% (equivalente a quatro universidades) são caracterizadas como públicas (UCL, ETH Zurich, University of California, Berkeley e Imperial College London).

A Tabela 4, apresenta as quinze instituições que se destacam no indicador Ensino: 
Tabela 4: Principais Instituições segundo o fator Ensino

\begin{tabular}{ccccc}
\hline $\begin{array}{c}\text { Ranking } \\
\text { Geral }\end{array}$ & $\begin{array}{c}\text { Ranking } \\
\text { Ensino }\end{array}$ & Universidade & País & Pontuação \\
\hline 4 & 1 & Stanford University & Estados Unidos & 92.8 \\
2 & 2 & California Institute of Technology & Estados Unidos & 92.1 \\
8 & 3 & Yale University & Estados Unidos & 92.0 \\
3 & 4 & Rniversity of Cambridge & Reino Unido & 91.4 \\
5 & 5 & Massachusetts Institute of Technology & Estados unidos & 90.5 \\
1 & 6 & University of Oxford & Reino Unido & 90.5 \\
6 & 7 & Princeton University & Estados Unidos & 90.3 \\
7 & 8 & Harvard University & Estados Unidos & 89.2 \\
9 & 9 & University of Chicago & Estados Unidos & 89.1 \\
24 & 10 & Peking University & China & 89.1 \\
11 & 11 & University of Pennsylvania & Estados Unidos & 87.5 \\
23 & 12 & Tsinghua University & China & 86.6 \\
36 & 13 & The University of Tokyo & Japão & 85.9 \\
16 & 14 & Columbia University & Estados Unidos & 85.6 \\
10 & 15 & Imperial College London & Reino Unido & 84.5 \\
\hline
\end{tabular}

Fonte: Adaptado de Times Higher Education (2019)

Nota-se uma mudança de distribuição geográfica das universidades quanto ao fator Ensino, apesar de $60 \%$ das instituições ainda serem estadunidenses, seguidos de $20 \%$ britânicas, 13,34\% chinesas e 6,66\% japonesas. Verificou-se que a Stanford University, que classificou-se na melhor posição, apresenta em sua estratégia, a aplicação de metodologias de ensino tanto tradicionais quando ativas, de acordo com o contexto do curso e da disciplina utilizada, adaptando os melhores métodos para melhor desenvolvimento dos alunos (STANFORD UNIVERSITY, 2019), o que pode corroborar com a avaliação da universidade na pesquisa de reputação, visto que esta métrica representa $15 \%$ do total do indicador.

A segunda colocada, California Institute of Technology (Caltec), apresenta um programa visando a melhoria da efetividade do ensino, que promove o relacionamento entre alunos de pós-graduação e pós-doutorado, com alunos de graduação, impulsionando as técnicas de ensino da instituição e fomentando a pesquisa nos primeiros anos de formação. Além do programa Summer Undergraduate Research Fellowships (SURF), que gera colaboração entre seus estudantes de graduação e especialistas de mercado para o desenvolvimento de projetos em seus campos de conhecimento (CALTECH, 2019).

A Yale University tem entre suas estratégias de ensino, o uso de metodologias ativas, sendo estas adaptadas e aplicadas de acordo com os cursos e as disciplinas. Entre as metodologias centradas no aluno que a universidade utiliza a sala de aula invertida, o ensino com base em estudos de caso e o uso de tecnologias digitais (YALE, 2019).

A distribuição geográfica das mais bem ranqueadas quanto ao Ensino é apresentada na Figura 3. 
Figura 3: Distribuição Geográfica das melhores Instituições ranqueadas, segundo o fator Ensino

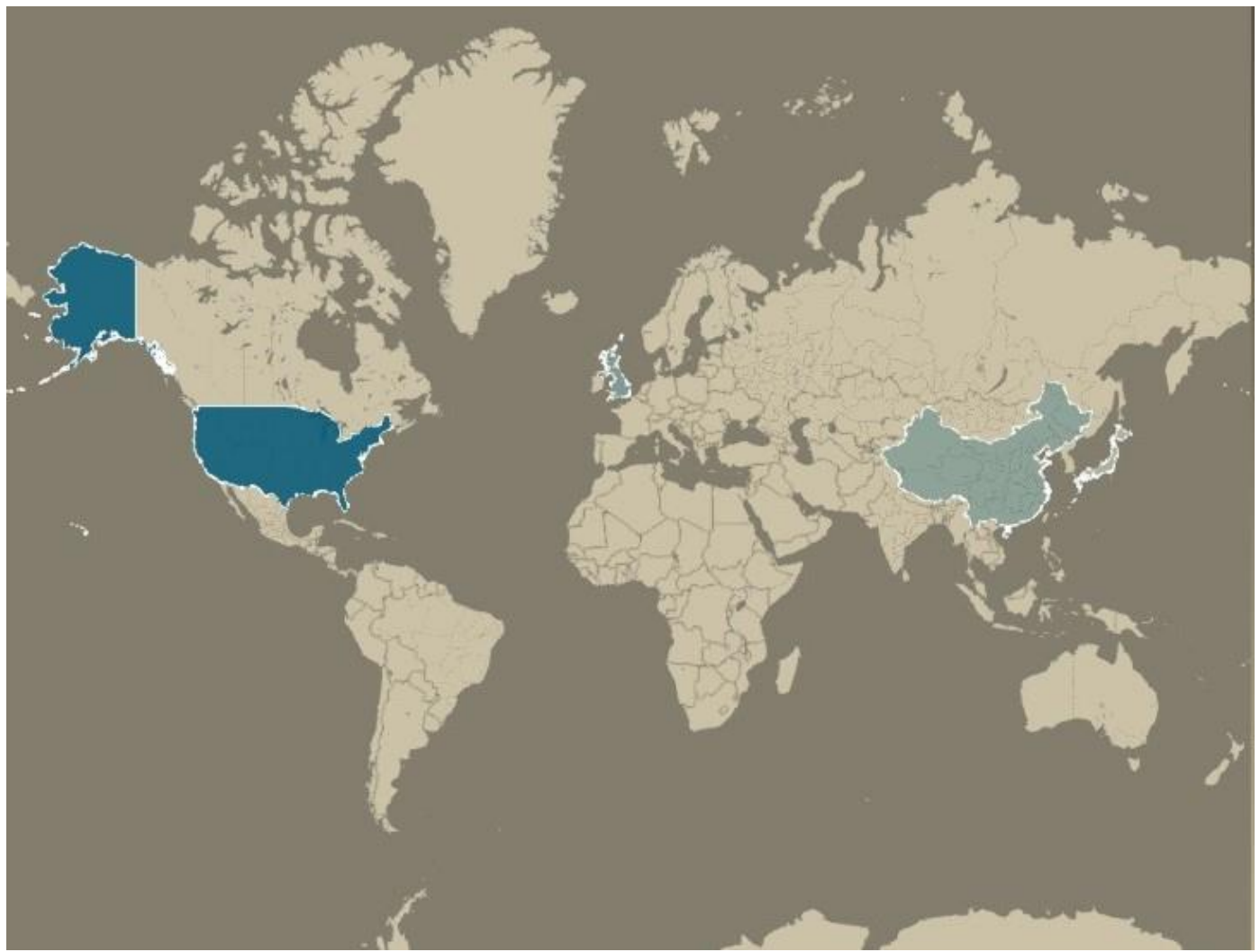

Fonte: Autores (2019)

Nesse fator, destacam-se três universidades asiáticas, que no ranking geral estariam na vigésima-quarta (Peking University), vigésima terceira (Tsinghua University) e trigésima sexta (The University of Tokyo) posições. Infere-se que estas universidades, apesar de não atingirem melhor classificação no ranking geral, apresentaram melhor resultados considerando apenas o indicador Ensino.

A Peking University passou por reformas políticas em seu sistema de ensino nos últimos anos, buscando atender necessidades particulares de seus discentes, além das lições dadas em sala de aula (PEKING UNIVERSITY, 2019). Quanto a Universidade de Tsinghua, identificou-se a política de educação continuada como sua principal estratégia de ensino (TSINGHUA UNIVERSITY, 2019).

A universidade de Tokyo, por sua vez, utiliza metodologias tradicionais de ensino, porém, garante investimentos para a abertura de novos cursos, focados no ensino e uma nova faculdade que tem como base estudos na área da Educação (THE JAPAN TIMES, 2019; UNIVERSITY OF TOKIO, 2019).

A Tabela 5 classifica as instituições que se destacam no indicador "Pesquisa".

Tabela 5: Principais Instituições segundo fator Pesquisa

\begin{tabular}{ccccc}
\hline $\begin{array}{c}\text { Ranking } \\
\text { Geral }\end{array}$ & $\begin{array}{c}\text { Ranking } \\
\text { Pesquisa }\end{array}$ & Universidade & País & Pontuação \\
\hline 1 & 1 & University of Oxford & Reino Unido & 99.6 \\
3 & 2 & University of Cambridge & Reino Unido & 98.7 \\
7 & 3 & Harvard University & Estados Unidos & 98.6 \\
2 & 4 & California Institute of Technology & Estados Unidos & 97.2 \\
4 & 5 & Stanford University & Estados Unidos & 96.4 \\
6 & 6 & Princeton University & Estados Unidos & 96.3 \\
8 & 7 & Yale University & Estados Unidos & 94.8 \\
23 & 8 & Tsinghua University & China & 94.0 \\
13 & 9 & ETH Zurich & Suiça & 92.8 \\
5 & 10 & Massachusetts Institute of Technology & Estados Unidos & 92.4 \\
9 & 11 & University of Chicago & Estados Unidos & 91.4
\end{tabular}




\begin{tabular}{ccccc}
12 & 12 & Johns Hopkins University & Estados Unidos & 91.4 \\
13 & 13 & University of California, Berkeley & Estados Unidos & 90.6 \\
25 & 14 & National University of Singapore & Cingapura & 90.4 \\
11 & 15 & University of Pennsylvania & Estados Unidos & 90.4 \\
\hline
\end{tabular}

Fonte: Adaptado de Times Higher Education (2019)

A Tabela 5, evidencia que as melhores universidades segundo o fator "Pesquisa", são as universidades de Oxford, seguida por Cambridge e Harvard. Entretanto, as instituições estadunidenses continuam sendo maioria $(66,67 \%)$, seguidas das britânicas $(13,33 \%)$ e uma representante suíça, chinesa e cingapuriana (cada uma com cerca de $6,66 \%$ do total.

As três instituições melhores classificadas apresentam programas de apoio à pesquisa de acordo com os cursos que oferecem, sendo que as pesquisas podem surgir internamente, ou solicitadas por empresas e indústrias, sendo que a grande maioria é financiada pelo setor privado (CAMBRIDGE, 2020; HARVARD, 2020; OXFORD, 2020). A universidade de Harvard (2020), cita que a instituição recebe aproximadamente 800 milhões de dólares por ano para financiamento de pesquisas.

A distribuição segundo o fator Pesquisa é o que mais se assemelha ao ranking geral, tendo Oxford como líder em ambas. No entanto, o indicador contou com uma distribuição geográfica com mais países representados do que no ranking geral. Tal distribuição é apresentada na Figura 4.

Figura 4: Distribuição Geográfica das Instituições mais bem ranqueadas segundo o fator pesquisa

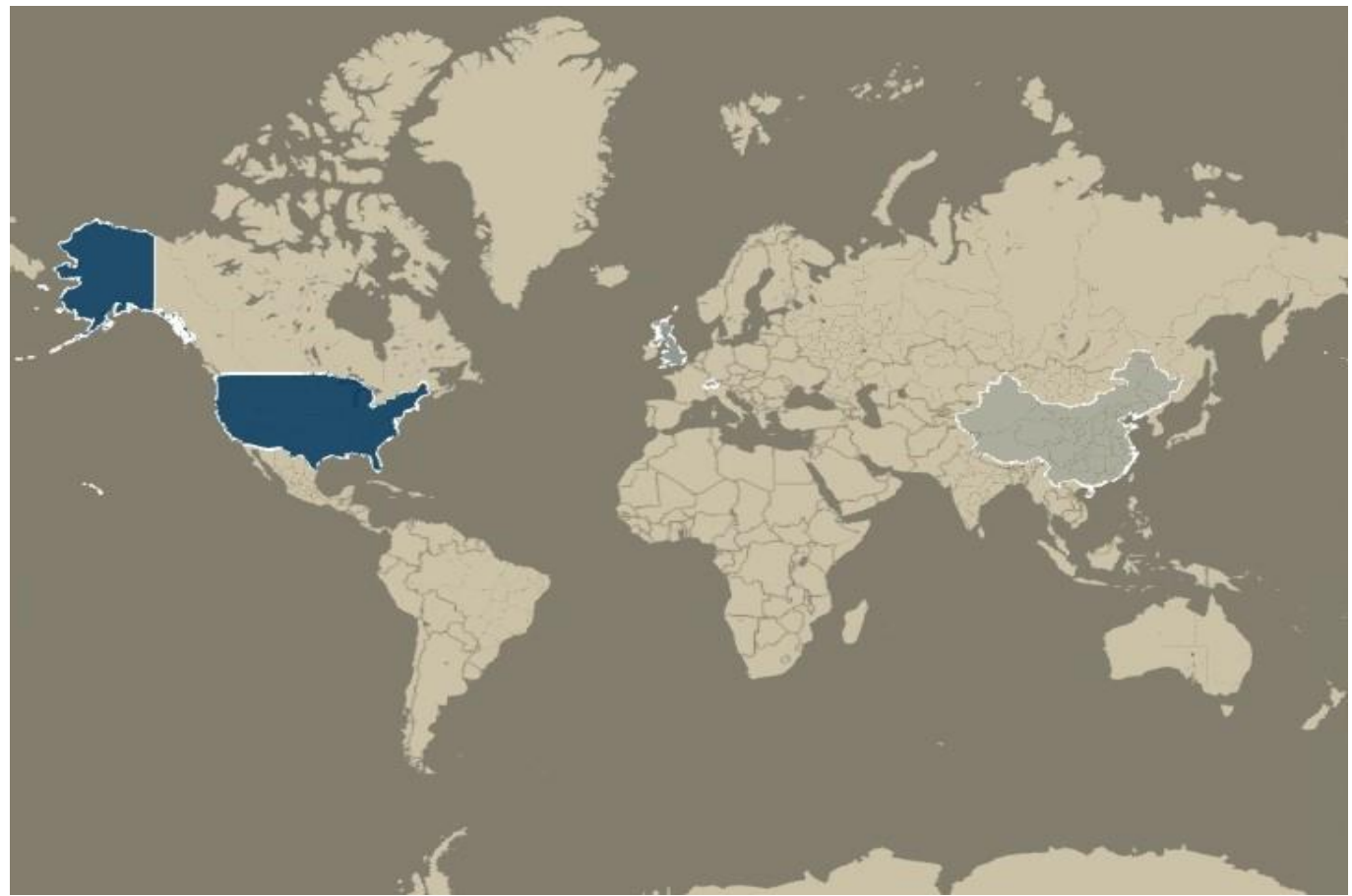

Fonte: Autores (2019)

É possível visualizar, através da Figura 4, um maior número de países que compõe as quinze melhores colocações, sendo 10 instituições estadunidenses, duas do Reino Unido, uma chinesa, uma de Singapura e uma da Suíça.

As universidades Oxford, Cambridge, Harvard, Caltech, Stanford, Princeton, Yale, Tsinghua, MIT, Chicago e Pennsylvania se fazem presentes entre as quinze melhor tanto no fator ensino, quanto no fator pesquisa, sendo que a ETH Zurique, a Universidade John Hopkins, a Universidade da California (Berkley) e a Universidade Nacional de Cingapura não se encontram em destaque no indicador do ensino. 
O envolvimento de alunos de graduação em pesquisas científicas, pode impactar para a hegemonia das universidades estadunidenses. Hu, Ku e Gayles (2007), citam que, a inclusão de graduandos em atividades de pesquisa cientifica, foi pauta das reformas do ensino superior nos Estados Unidos, realizadas entre 2002 e 2006.

Por fim, os dados referentes ao indicador "renda industrial" se apresentam da forma de com a Tabela 6.

Tabela 6: Principais Instituições segundo o fator renda industrial

\begin{tabular}{|c|c|c|c|c|}
\hline $\begin{array}{l}\text { Ranking } \\
\text { Geral }\end{array}$ & $\begin{array}{l}\text { Ranking } \\
\text { Ensino }\end{array}$ & Universidade & País & Pontuação \\
\hline $1001+$ & 1 & Anadolu University & \multirow{2}{*}{$\begin{array}{l}\text { Turquia } \\
\text { Taiwan }\end{array}$} & 100.0 \\
\hline $\begin{array}{l}801- \\
1000\end{array}$ & 2 & Asia University, Taiwan & & 100.0 \\
\hline $\begin{array}{c}86 \\
601-800\end{array}$ & $\begin{array}{l}3 \\
4\end{array}$ & $\begin{array}{l}\text { University of Freiburg } \\
\text { Istanbul Technical University }\end{array}$ & \multirow{2}{*}{$\begin{array}{c}\text { Alemanha } \\
\text { Turquia } \\
\text { Emirados Arabes } \\
\text { Unidos }\end{array}$} & $\begin{array}{l}100.0 \\
100.0\end{array}$ \\
\hline $351-400$ & 5 & Khalifa University & & 100.0 \\
\hline 32 & 6 & LMU Munich & \multirow{2}{*}{$\begin{array}{l}\text { Alemanha } \\
\text { Russia }\end{array}$} & 100.0 \\
\hline $401-500$ & 7 & $\begin{array}{c}\text { National Research Nuclear University } \\
\text { MEPhl }\end{array}$ & & 100.0 \\
\hline 43 & 8 & Technical University of Munich & Alemanha & 100.0 \\
\hline 23 & 9 & Tsinghua University & China & 100.0 \\
\hline 59 & 10 & Wageningen University \& Research & Países Baixos & 100.0 \\
\hline 194 & 11 & University of the Witwatersrand & Africa do Sul & 100.0 \\
\hline 107 & 12 & Zhejiang University & China & 100.0 \\
\hline 20 & 13 & Duke University & Estados Unidos & 99.9 \\
\hline 110 & 14 & $\begin{array}{c}\text { Korea Advanced Institute of Science } \\
\text { and Technology (KAIST) }\end{array}$ & Coréia do Sul & 99.9 \\
\hline $201-250$ & 15 & $\begin{array}{c}\text { Moscow Institute of Physics and } \\
\text { Technology }\end{array}$ & Russia & 99.9 \\
\hline
\end{tabular}

Fonte: Adaptado de Times Higher Education (2019)

Quanto ao resultado das quinze IES mais bem ranqueadas segundo o indicador "renda industrial", nota-se uma grande dissemelhança em relação as classificações anteriores. Os resultados mostram que as quinze principais universidades classificadas no ranking geral, que se repetiram tanto no indicador Ensino quanto na Pesquisa, não estão presentes nesse indicador. Isso pode ser resultado da hegemonia dos indicadores ensino e pesquisa nestas IES, e de que o relacionamento com o mercado é ainda incipiente nas organizações universitárias, sendo que parte das inovações e pesquisas geradas dentro do ambiente acadêmico, ainda encontra barreiras para encontrar parceiros para a comercialização ou aplicação na indústria (DIAS; PORTO, 2018). As Instituições mais bem ranqueadas neste indicador são $20 \%$ de origem alemã, 13,33\% turcas, 13,33\% russas, $13,33 \%$ chinesas e cerca de $6,66 \%$ para cada um dos seis países que apresentam uma instituição.

A única universidade que se classificou entre as quinze melhores, segundo os três fatores avaliados é a Universidade de Tsinghua, apesar de estar na vigésima-terceira posição do ranking geral.

A universidade de Tsinghua, faz parte de uma estratégia do governo chinês para estabelecer WCU's dentro de seu território nacional. Como resposta, o foco da instituição é atender diretamente as três missões universitárias (ensino, pesquisa e transferência de tecnologia), não priorizando parcerias internacionais, fator este que pode ser determinante para uma melhor classificação no ranking geral do THE-WU (YANG; WELCH, 2011).

O ranking específico do indicador "renda industrial", apresenta maior diversidade geográfica quando comparado aos rankings anteriores, conforme é apresentado na Figura 5 . 
Figura 5: Distribuição Geográfica das Instituições mais bem ranqueadas segundo o fator renda industrial

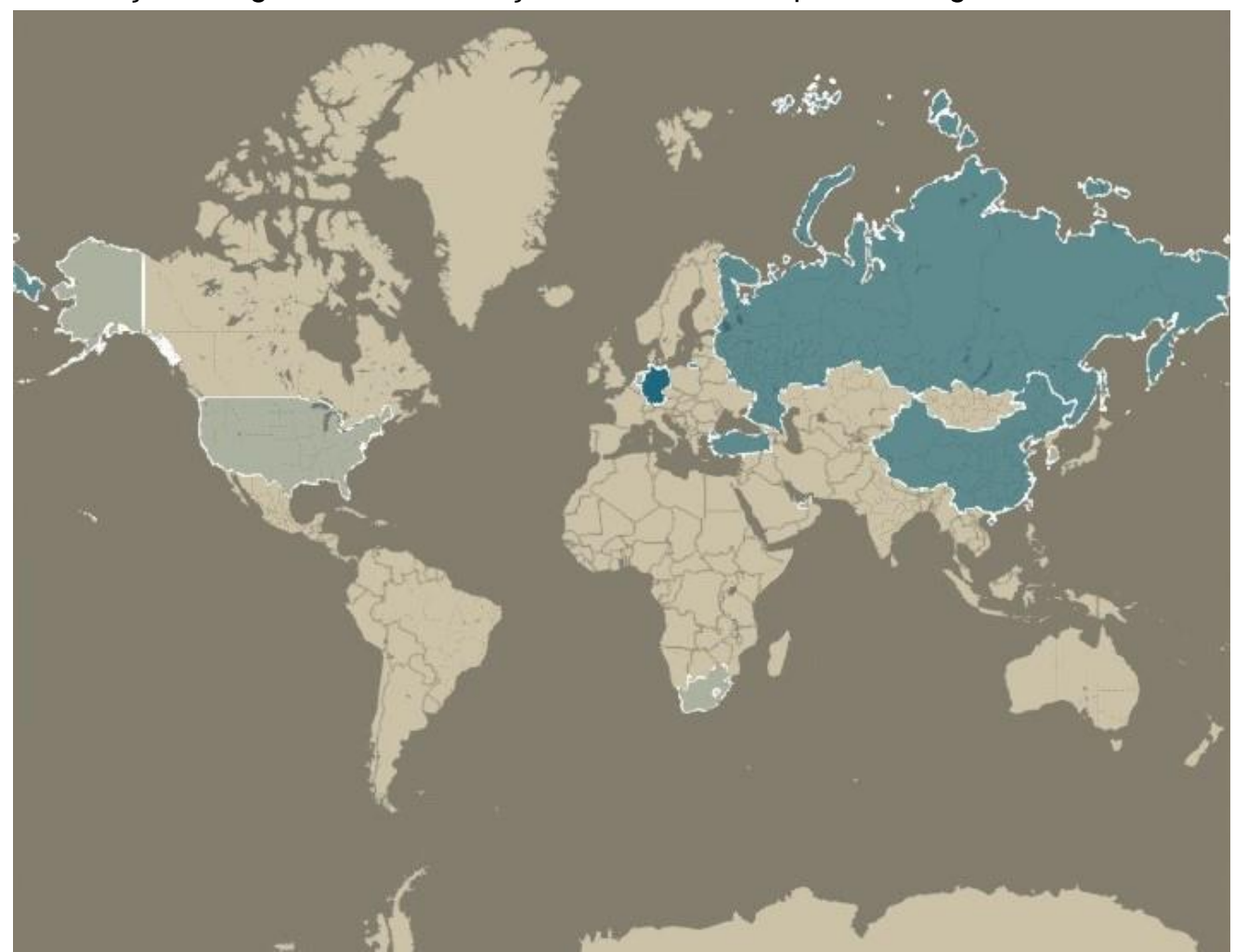

Fonte: Autores (2019)

Evidencia-se um total de dez países representados no ranking de renda industrial, sendo os três primeiros colocados de origem Turca, Taiwanesa e Alemã. As universidades Turcas, segundo Reisman, Caspar e Aktas (2004), historicamente priorizam a transferência de tecnologia entre suas missões, sendo inclusive uma questão cultural. Yu e Yeh (2016), evidenciam como o governo taiwanês utiliza técnicas de transferência de tecnologia juntamente as IES para sanar as lacunas tecnológicas e de mercado apresentadas no país.

Quanto as instituições alemãs, Grimpe e Fier (2009) citam que, os pesquisadores possuem maior foco em atividades formais de transferência de tecnologia, capazes de produzir boas pesquisas científicas e patentes derivadas destas pesquisas.

Após análise, pode-se inferir que os indicadores Ensino e Pesquisa apresentaram uma centralização das IES dentro do eixo Estados Unidos e Reino Unido, sugerindo que as instituições localizadas nesses países são referências em seus campos de atuação. Porém, quando se trata de transferência de tecnologia das universiddaes para o mercado, correlacionado ao fator Renda Industrial, a distribuição geográfica das Instituições mais bem posicionadas no ranking foge desse eixo. Verificou-se que as universidades turcas, alemãs, e chineses apresentaram maior destaque neste quesito, mostrando que além da Alemanha, a Ásia está atuando de maneira expressiva no relacionamento das IES com o mercado.

Os resultados revelam que ainda há uma priorização dos rankings na avaliação de métricas direcionadas ao Ensino e a Pesquisa, favorecendo universidades que já atuam fortemente nestes campos, e uma certa negligência com métricas associadas a terceira missão, citada por Montesinos et al. (2008), referente a Transferência de Tecnologia. Isso corrobora com a pesquisa de Perez-Esparrels e Orduna-Malea (2018), que criticam a baixa visibilidade de algumas instituições que são afetadas na classificação do THE, visto que o ranking ainda não considera critérios como comercialização de tecnologia, patentes e criação de startups, indicadores estes ligados a instituições com um caráter mais técnico e empreendedor. 
As análises também mostraram que as principais universidades do eixo Estados Unidos e Reino Unido presentes no ranking, são instituições semi-privadas ou privadas, que captam recursos de diversos meios além dos repasses governamentais, permitindo uma maior autonomia financeira e de alocação de recursos para as instituições (DOBBINS; KNILL; VOGTLE, 2019). Segundo Estermann (2015), a autonomia é necessária para que as instituições atendam à suas missões da forma que considerem mais eficiente, sendo necessário apenas aprovações internas quanto as distribuições de recurso e possuindo uma melhor performance nos rankings em relação as instituições que dependem de políticas públicas.

\section{Conclusão}

Por meio dos resultados foi possível identificar que há uma discrepância entre os rankings específicos de ensino, pesquisa e renda industrial. Quanto aos dois primeiros indicadores analisados, onze universidades se repetiram como uma das quinze melhores ranqueadas no indicador global, sendo estas, oito de origem estadunidense, duas do Reino Unido e apenas uma chinesa. Enquanto para o indicador de "renda industrial" apenas a universidade de Tsinghua se repete, estando presente nos três indicadores analisados, Ensino, Pesquisa e Renda Industrial.

Conclui-se que a universidade de Tsinghua é a única instituição dentre as quinze selecionadas, que melhor atende as três missões: de ensinar, pesquisar e transferir tecnologia para a sociedade, embora classifique-se apenas na vigésima-terceira posição do ranking geral THE-WU 2020.

Infere-se que a autonomia financeira das instituições pode ser determinante para a classificação das universidades, segundo os rankings, sendo que cada um dos fatores de análise leva em consideração uma série de critérios específicos, que podem ser abordados de diversas formas e que dependem da distribuição de recursos nas instituições. No caso das instituições públicas (como Tsinghua) as políticas públicas são determinantes, pois necessitam do fomento e de regulamentação do Estado.

Como sugestão para futuros trabalhos poderiam ser avaliadas as questões de "reconhecimento internacional" das instituições ranqueadas no THE, a fim de correlacionar os resultados com marketing e networking destas IES.

\section{Referências}

AL KUWAITI, Ahmed; DOWNING, Kevin; SUBBARAYALU, Arun Vijay. Performance of Saudi Universities in Global Rankings and appropriate strategies for its improvement. Library Philosophy and Practice, p. 1-21, 2019.

BASTEDO, Michael N.; BOWMAN, Nicholas A. US News \& World Report college rankings: Modeling institutional effects on organizational reputation. American Journal of Education, v. 116, n. 2, p. 163-183, 2009.

BATY, Phil. The times higher education world university rankings, 2004-2012. Ethics in Science and Environmental Politics, v. 13, n. 2, p. 125-130, 2014.

CALTECH. Center for Teaching, Learning \& Outreach (CTLO): CPET Grad / Postdoc Community. Disponível em: <http://ctlo.caltech.edu/universityteaching/programs/cpet>. Acesso em: 28 nov. 2019. 
CAMBRIDGE. Research: Research at Cambridge. 2020. Disponível em: $<$ https://www.cam.ac.uk/research/research-at-cambridge>. Acesso em: 07 jan. 2020.

CHENG, Ying; WANG, Qi; LIU, Nian Cai. How world-class universities affect global higher education. In: How World-Class Universities Affect Global Higher Education. Sense Publishers, Rotterdam, 2014. p. 1-10.

DE DOMINICIS, Laura; PÉREZ, Susana Elena; FERNÁNDEZ-ZUBIETA, Ana. European university funding and financial autonomy. European Union, 2011.

DEEM, Rosemary; MOK, Ka Ho; LUCAS, Lisa. Transforming higher education in whose image? Exploring the concept of the 'world-class' university in Europe and Asia. Higher education policy, v. 21, n. 1, p. 83-97, 2008.

DELGADO-MÁRQUEZ, Bianca L.; HURTADO-TORRES, Nuria Esther; BONDAR, Yaroslava. Internationalization of higher education: Theoretical and empirical investigation of its influence on university institution rankings. International Journal of Educational Technology in Higher Education, v. 8, n. 2, p. 265-284, 2011.

DOBBINS, Michael; KNILL, Christoph; VÖGTLE, Eva Maria. An analytical framework for the cross-country comparison of higher education governance. Higher education, v. 62, n. 5, p. 665-683, 2011.

DOĞAN, Güleda; AL, Umut. Is it possible to rank universities using fewer indicators? A study on five international university rankings. Aslib Journal of Information Management, v. 71, n. 1, p. 18-37, 2019.

ESTERMANN, Thomas. University autonomy in Europe. University Education, n. 3. O. 28- 32. 2015.

ETZKOWITZ, Henry et al. The future of the university and the university of the future: evolution of ivory tower to entreprene

urial paradigm. Research policy, v. 29, n. 2, p. 313-330, 2000.

FORSTER, Nick. Why are there so few world-class universities in the Middle East and North Africa?. Journal of Further and Higher Education, v. 42, n. 8, p. 1025-1039, 2018.

GRIMPE, Christoph; FIER, Heide. Informal university technology transfer: a comparison between the United States and Germany. The Journal of Technology Transfer, v. 35, n. 6, p. 637-650, 2010.

HARVARD. Research. 2020. Disponível em: <https://www.harvard.edu/oncampus/research>. Acesso em: 07 jan. 2020.

HAYES, Aneta. Why international students have been "TEF-ed out"?. Educational Review, v. 69, n. 2, p. 218-231, 2017.

HEYNEMAN, Stephen P.; LEE, Jeongwoo. World-class universities: The sector requirements. In: Institutionalization of World-Class University in Global Competition. Springer, Dordrecht, 2013. p. 45-58. 
JEREMIC, Veljko; JOVANOVIC-MILENKOVIC, Marina. Evaluation of Asian university rankings: position and perspective of leading Indian higher education institutions. Current Science, p. 1647-1653, 2014.

KROTH, Anna; DANIEL, H.-D. Internationale Hochschulrankings. Zeitschrift für Erziehungswissenschaft, v. 11, n. 4, p. 542, 2008.

LAUGHTON, David. Why was the QAA approach to teaching quality assessment rejected by academics in UK HE? Assessment \& evaluation in higher education, v. 28, n. 3, p. 309-321, 2003.

LIU, Zhimin; MOSHI, Goodluck Jacob; AWUOR, Cynthia Mwonya. Sustainability and Indicators of Newly Formed World-Class Universities (NFWCUs) between 2010 and 2018: Empirical Analysis from the Rankings of ARWU, QSWUR and THEWUR. Sustainability, v. 11, n. 10, p. 2745, 2019.

MARGINSON, Simon. Global university rankings: Implications in general and for Australia. Journal of Higher Education Policy and Management, v. 29, n. 2, p. 131-142, 2007.

MARGINSON, Simon; VAN DER WENDE, Marijk. To rank or to be ranked: The impact of global rankings in higher education. Journal of studies in international education, v. 11, n. 3-4, p. 306-329, 2007.

MARGINSON, Simon. University rankings and social science. European Journal of Education, v. 49, n. 1, p. 45-59, 2014.

OLSSEN, Mark; PETERS, Michael A. Neoliberalism, higher education and the knowledge economy: From the free market to knowledge capitalism. Journal of education policy, $v$. 20, n. 3, p. 313-345, 2005.

MONTESINOS, Patricio et al. Third mission ranking for world class universities: Beyond teaching and research. Higher education in Europe, v. 33, n. 2-3, p. 259-271, 2008.

OXFORD. Research. 2020. Disponível em: <https://www.ox.ac.uk/research?wssl=1>. Acesso em: 07 jan. 2020.

PEREZ-ESPARRELLS, Carmen; ORDUNA-MALEA, Enrique. Do the technical universities exhibit distinct behaviour in global university rankings? A Times Higher Education (THE) case study. Journal of Engineering and Technology Management, v. 48, p. 97-108, 2018.

REISMAN, Arnold; CAPAR, Ismail; AKTAS, Emel. Turkey's Development: The Role of Technology Transfer. Available at SSRN 607841, 2004.

RAHIM, Rahimin Affandi Abdul; MAN, Saadan; ABDULLAH, Luqman Haji. Transformation of Shariah Studies in Malaysian Higher Learning Institution. Middle-East Journal of Scientific Research, v. 12, n. 2, p. 145-154, 2012. 
RODIONOV, Dmitriy Grigorievich; RUDSKAIA, Irina Andreevna; KUSHNEVA, Olga Alexandrovna. The importance of the university world rankings in the context of globalization. Life Science Journal, v. 11, n. 10, p. 442-446, 2014.

ROSSELOT, J. et al. La globalización en la educación superior europea. Pródromos para nuestra educación médica: The precursor of changes in Chilean medical education. Revista médica de Chile, v. 133, n. 7, p. 833-840, 2005.

SABATA, Martí Parellada; CARDEÑOSA, Montserrat Álvarez. Reputación y" rankings". Debats, v. 131, n. 2, p. 15-26, 2017.

SILVA, Lidiane Rodrigues Campêlo da et al. PESQUISA DOCUMENTAL:: ALTERNATIVA INVESTIGATIVA NA FORMACÃO DOCENTE. In: CONGRESSO NACIONAL DE EDUCAÇÃO - EdUCERE, 9., 2009, Curitiba. Anais... Paraná: Pucpr, 2009. p. 4554 4566.

$\mathrm{SOH}$, Kaycheng. Times Higher Education 100 under 50 ranking: old wine in a new bottle?. Quality in Higher education, v. 19, p. 111-121, 2013.

STANFORD UNIVERSITY. Teaching Commons: Teaching Strategies. 2019. Disponível em: $\quad<$ https://teachingcommons.stanford.edu/resources/teaching-resources/teachingstrategies>. Acesso em: 28 nov. 2019.

TELEGRAPH REPORTERS. Oxford named as best university in the world, new rankings show. $2019 . \quad$ Disponível em: $<$ https://www.telegraph.co.uk/news/2019/09/11/oxford-named-best-university-world-newrankings-show/>. Acesso em: 28 nov. 2019.

THE JAPAN TIMES. Improving teaching at universities. 2019. Disponível em: $<$ https://www.japantimes.co.jp/opinion/2013/04/16/editorials/improving-teachng-atuniversities/\#.XeE-i-hKizc>. Acesso em: 28 nov. 2019.

THIEN, Lei Mee; ONG, Mei Yean. The applicability of course experience questionnaire for a Malaysian university context. Quality Assurance in Education, v. 24 p.41-55, 2016.

TIMES HIGHER EDUCATION. World University Rankings 2019. 2019. Disponível em: $<$ https://www.timeshighereducation.com/world-university-rankings/2019/worldranking\#!/page/0/length/25/sort by/rank/sort order/asc/cols/stats >. Acesso em: 25 nov. 2019.

TIMES HIGHER EDUCATION. World University Rankings 2019: methodology. 2019. Disponível em: <https://www.timeshighereducation.com/world-university-rankings/worlduniversity-rankings-2019-methodology>. Acesso em: 25 nov. 2019.

TSINGHUA UNIVERSITY. Continuing Education. 2019. Disponível em: $<$ https://www.tsinghua.edu.cn/publish/thu2018en/newthuen cnt/02-admissions-6.html>. Acesso em: 28 nov. 2019. 
UNIVERSITY OF TOKIO. Academics: Faculty of Education. 2019. Disponível em: $<$ https://www.u-tokyo.ac.jp/en/academics/facultyofeducation.html>. Acesso em: 28 nov. 2019.

VALMORBIDA, Sandra MI; CARDOSO, Thuine L.; ENSSLIN, Sandra Rolim. Rankings universitários: análise dos indicadores utilizados. Sociedade, Contabilidade e Gestão, v. 10, n. 2, 2015.

WONG, Poh; SINGH, Annette. University patenting activities and their link to the quantity and quality of scientific publications. Scientometrics, v. 83, n. 1, p. 271-294, 2009.

YALE. Poorvu Center for Teaching and Learning: Strategies for Teaching. 2019. Disponível em: <https://poorvucenter.yale.edu/FacultyResources/Teaching-Strategies>. Acesso em: 28 nov. 2019

YU, Hui-Yun; YEH, Kuang S. Technology transfer in Taiwan's information industry: The lessons. Research-Technology Management, v. 39, n. 5, p. 26-30, 1996. 